\section{Adaptive and non-adaptive suicide in aphids}

SIR - McAllister and Roitberg' recently reported evidence for "adaptive suicide" in pea aphids (Acyrthosiphon pisum). The aphids were said to have three strategies of escape from predators: backing-up, running, and falling from the food plant. Parasitized aphids from dry regions used the falling strategy more often than expected and this was put forward as suicidal behaviour.

The authors attempted to exclude the possibility that the apparently suicidal behaviour was a direct maladaptive effect of the parasite: they compared the behaviour of aphids from coastal and from dry habitats, where the fitness consequences of falling were different. However, the experimental rationale relies upon the unfounded assumption that the two aphid populations were genetically homogeneous at loci not involved in antipredator behaviour. Indeed, in a previous paper $^{3}$, it is implicitly stated that aphids living in two areas, coastal and dry, "are considered different biotypes". Therefore, aphids from the dry habitat may simply be more susceptible to the direct effects of the parasite.

The aphids used were only parasitized by wasps from their own habitat. Therefore, the observed differences between aphid populations may have been a function of the parasites rather than the hosts. A standardized experiment using all four host/parasite combinations could be performed to examine this possibility.

The increased falling behaviour of parasitized aphids from hot regions may simply be a superior means of escape from predators. The authors give an imprecise indication of the fitness consequences of different escape strategies in each of the two aphid habitats. Moreover, they do not indicate whether the fitness consequences differ between parasitized and unparasitized individuals.

The conclusion that suicide is adaptive will apply only if it is under genetic control. The authors have not shown this. Crosses between the two aphid populations are needed.

Following evolutionary stable strategy theory ${ }^{4}$, it is likely that all three escape strategies have equal payoffs at equilibrium, despite having apparently different fitness consequences. Parasitized individuals could commit suicide by performing any escape strategy in excess. Perhaps the dropping strategy is most efficient for suicide in hot areas, but this does not explain the absence of suicidal behaviour in the coastal population. The fitness of parasitized individuals from the temperate coastal region would still be lowered if an excess chose to jump rather than perform an optimal mixture of strategies.
Why is suicide so inefficient? It is curious that the parasitized aphids committed suicide only when approached by a ladybird, or when escape pheromones had been detected. More obvious means of committing suicide would simply be not to eat or to fall from the food plant spontaneously. It was proposed that the suggested suicide mechanism evolved because it was an easy modification of an existing behavioural strategy. Even so, the proposed modification of behaviour is surprisingly slight.

Finally, it is not in the interest of parasites that their hosts should commit suicide. Natural selection on parasites may favour the subversion of the host's suicidal behaviour'. In any ensuing 'arms race', asymmetries of selection should favour the parasite.

In my opinion, the evidence of McAllister and Roitberg does not give convincing support to the 'host suicide hypothesis'. Their results are compatible with a number of other hypotheses, both adaptive and non-adaptive.

Department of Genetics, IAN TOMLINSON

University of Cambridge,

219D Huntingdon Road,

Cambridge CB3 ODL, UK

McAllister, M.K. \& Roitberg, B.D. Nature 328, 797-799 (1987)

2. Smith Trail, D.R. Am. Nat. 116, 77-91 (1981)

3. Roitberg, B.D. \& Myers, J.H. Can. Ent. 111, 515-519 (1979).

4. Maynard Smith, J. Evolution and the Theory of Games (Cambridge University Press, 1982).

5awkins, R. The Extended Phenotype (Freeman, Oxford, 1982).

SIR-I wish to question the reasoning behind a recent paper by McAllister and Roitberg ${ }^{1}$, intended to demonstrate apparently suicidal behaviour in parasitized pea aphids from British Columbia. Pea aphids were chosen because of their parthenogenetic mode of reproduction and low dispersal rate - both factors conducive to verification of the 'host suicide hypothesis', which holds that a host whose reproductive potential has effectively been reduced to zero by a parasitoid may yet enhance its inclusive fitness ${ }^{3}$ by actively increasing the probability of death, so preventing maturation of the endoparasite and preventing its spread among closely aggregated clonal kin.

McAllister and Roitberg argue that pea aphids in a moist climate stand a reasonable chance of survival on the ground, whereas pea aphids from a hot, dry climate quickly die of heat stress and desiccation. In other words, under normal circumstances, an aphid from a dry climate would do better in terms of its individual fitness to remain on the leaf and back up when approached by a predator than to drop from the leaf - both of which are possible escape behaviours used by aphids $^{4}$. If the host suicide hypothesis holds, then parasitized aphids from a dry climate could enhance their inclusive fitness by increased dropping under the stimulus of a predator, while parasitized aphids from a moist climate would not increase the probability of death by dropping and so their escape behaviour would not alter when parasitized. The reported experiment seemed to confirm these predictions.

The difficulty I find with this argument is that, to increase its inclusive fitness, the host aphid need not commit suicide, but merely need isolate itself (along with its endoparasite) from its stationary aggregate of kin. That is, once the host has dropped, it makes no difference to the fitness of its kin (and hence its inclusive fitness) whether death on the ground is the result of this behaviour or not. In fact, as its aim is to increase the fitness of its kin relative to the fitness of non-kin ${ }^{5}$, it would do better to remain alive and so allow the parasitoid to mature, because mathematically the parasitoid is more likely to find its way to aggregates of non-kin than back to the original aggregate of kin.

On the basis of this 'host isolation hypothesis', I suggest that, as described in the report ${ }^{1}$, there should have been increased dropping of parasitized dry-climate aphids, but, contrary to the reported findings, there should have been no difference between the behaviour of dry-climate aphids and moist-climate aphids. Both should have increased dropping behaviour when parasitized.

I am at a loss to explain the apparent success of the reported experiment. But, where a premise is in error, agreement between prediction and observation cannot be taken as confirmation of the original hypothesis.

\section{Division Street,}

Blair Latta

\section{Kingston, Ontario,}

\section{K7L 3M2 Canada}

1. McAllister, M.K. \& Roitberg, B.D. Nature 328, 797-799 (1987).

2. Smith Trail, D.R. Am. Nat. 116, 77-91 (1981).

3. Hamilton, W.D. J. theor. Biol. 7, 1-52 (1964)

4. Brodsky, L.M. \& Barlow, C.A. Can. J. Zool. 64, 937-939 (1986).

5. Lewontin, R.C. Sci. Am. 239(3), 213-230 (1978).

\section{Translational control in homoeobox mRNAs?}

SIR-The deduced sequences of several complete vertebrate homoeobox complementary (c)DNAs have recently been published. We have searched for regions conserved in genes from frogs ${ }^{1-4}$, mice ${ }^{5-7}$ and humans ${ }^{8,9}$ and have found extensive amino-acid conservation of X1Hbox 2 , HHO.cl, XHox-36 and Hox 1.1 throughout the whole coding region, such that they form a distinct family. The degree of similarity of X1Hbox 2 and HHO.cl suggests that these are the frog and human homologues of the same gene; in the mouse their homologue is called Hox $2.3^{10}$. 